\title{
Development and Evaluation of a Modular Energy Management Construction Kit
}

\section{Modulare Konfigurier- und Testplattform für die Untersuchung des Energiemanagements in selbsversorgten Anwendungen}

\author{
Jan Kokert, Tobias Beckedahl and L.M. Reindl \\ University of Freiburg, Department of Microsystems Engineering - IMTEK \\ Laboratory for Electrical Instrumentation, Georges-Köhler-Allee 106, 79110 Freiburg, Germany
}

\begin{abstract}
Our aim of research is to understand and to improve the power supply of energy-harvesting powered systems by means of finding the optimal energy management. In this paper we (1) propose a general energy management structure, (2) present a modular platform concept to realize various configurations of energy management, and (3) characterize management modules and prove the feasibility of the construction kit. The energy management components investigated are dc-dc converters, energy-supervisors, super capacitors and a dummy loads. The results show that all modules work properly and that interconnections has a negligible effect. Our tool offers a new approach to thoroughly investigate energy managements.
\end{abstract}

Keywords: Energy harvesting, energy management, modular platform, dc-dc converter, super capacitor

\section{Introduction}

Self-powered sensor nodes are electrical microsystems which continuously measure physical parameters of their surrounding. This might be the temperature, pressure, humidity [1], light intensity[2], vibration [3] or soil properties [4]. The wireless sensor nodes (WSN) send the data to the next node or to a central data collection unit for monitoring.

Batteries or energy harvesting $(\mathrm{EH})$ are used to supply power to the devices [5]. A battery has a limited lifetime and therefore needs to be replaced after a while which can cost more then the battery itself. EH utilizes the energy of the surrounding (no matter in which form) and converts it into electrical energy.

Micro-energy management (MEM) is essential to extract, transfer and regulate power from an energy harvester to a load, as shown in Fig. 1. It is a key component for self-powered sensor nodes and is thoroughly discussed in this article.

The remainder of the paper is organized as follows: in the state-of-the-art section we present energy managements of wireless sensor nodes and test

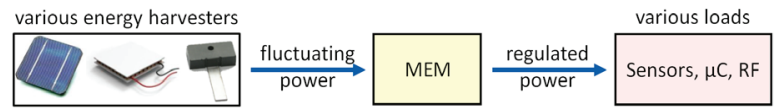

Fig. 1 General scenario in with a micro-energy management (MEM) supplies regulated power to a load from energy harvesting kits. After that wee will motivate our research approach in 3. We present a general structure of MEMs in 4 and our modular construction kit in 5 . In section 6 we show the evaluation of the kit components (modules and main board).

\section{State-of-the-art}

\subsection{WSNs which use Energy Management}

Below, we will present a couple of wireless sensor nodes (WSN) which implement a rather advanced energy management. All approaches make use of power converters (dc-dc) to increase the system efficiency and use intermediate storages to buffer power fluctuations. To give a better overview, we have summarized the issues and drawbacks first:

- The hardware of all systems presented is fixed on one printed circuit board (PCB), which makes varying different hardware components very impractical.

- Another issue in the presented work is that the leakage of super capacitors is measured and understood incorrectly. As [6] and [7] show, the voltage drop after charging is due to an internal charge redistribution, which we however, consider in our measurements.

- The structure of the energy managements presented so far is application specific and not generalized to cover all management configurations. 
The authors in [8] present a WSN prototype board powered by ten miniature PV cells and the bq25504 dc-dc converter. They realize a hybrid storage with a $5 \mathrm{~F}$ super capacitor and a $350 \mathrm{mAh}$ Li-lon Battery. The power management (decision making) is implemented in an CPLD. The board is comparably large $(18.8 \mathrm{~cm} \times 12.5 \mathrm{~cm})$ with a fixed management structure. Only jumpers offers to set different configurations. The power consumption of the CPLD and the involved analog-to-digital converters (ADCs) are not discussed.

In [9] the authors present an energy management with hybrid storage (super capacitor and Li-lon battery), bidirectional dc-dc converters and a $3.3 \mathrm{~V}$ dc-bus. The dc-dc converters are built using the TPS43000 dc-dc controller and external MOSFET transistors which makes it very versatile. The major drawback though, is the lack of configurations possible. Furthermore, it is not clear why the dc-bus is beneficial for the system.

The authors of [10] present an impedance matching for a thermoelectric generator (TEG) with a two stage concept, utilizing a Seiko chargepump and the TPS61020 boost converter. Again, the whole energy management structure is fixed which makes optimization very hard. Additionally, the supercap leakage current is misunderstood.

In [11] the author mentions a modular platform to evaluate different dc-dc converters and loads. All modules are connected in one string and the $32 \mathrm{l} / \mathrm{O}$ lines offers a very flexible usage. A drawback is the size of the modules $(16 \mathrm{~cm} \times 10 \mathrm{~cm})$ which are connected by huge $2 \times 32$ pin connectors.

\subsection{Test kits for Energy Management}

\subsubsection{Energy Harvesting Solution to go (WE)}

The German company Würth-Elektronik offers a commercial energy harvesting kit, which is shown

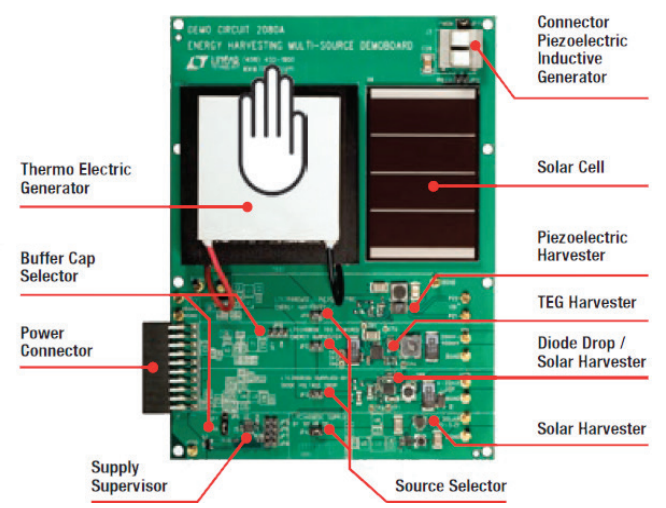

Fig. 2 Energy Harvesting Solution To Go which allows multi-source harvesting [www.we-online.de] in Fig. 2. The kit combines the EFM32 Giant Gecko Starter Kit from Silicon Labs with the Multi-Source Energy Harvester from Linear Technology and passive components, like coils, from Würth-Elektronik. Different dc-dc converters can be chosen by the jumpers, which only gives a little freedom of configuration. Multi-source harvesting is possible by using a combination of solar cell, thermoelectric generator (TEG) and the piezo harvester (for vibration). The kit provides no monitoring capability, except for the current measurement of the microcontroller.

\subsubsection{Energy Harvesting Platform (FE)}

A more versatile solution is the Energy Harvesting Platform from Future Electronics. Different harvesters, dc-dc converters, storages and sensors (loads) can be connected via a uniform interface to a main board. The structure however is rather fixed, including one harvester, two dc-dc converters, one storage and one load module. Further enhanced concepts like multi-source harvesting or a hybrid storage can not be realized. Furthermore, the main board includes fixed components: power paths are switched by bistable relays and the current is measured by fixed shunt resistors $(50 \mathrm{~m} \Omega)$.

\subsubsection{Vendor-specific EH demo boards}

Besides dc-dc IC evaluation boards some vendors also offer EH-demo boards which provide connectivity of different harvesters and loads and offer certain self monitoring capabilities. Worth to mention are the Analog Devices ADP5091 demo board, Maxim Integrated MAX17710 evaluation board, the enOcean EVA320-2 board and the IPS-EVAL-EH-01 evaluation board from Infinite Power Solutions.

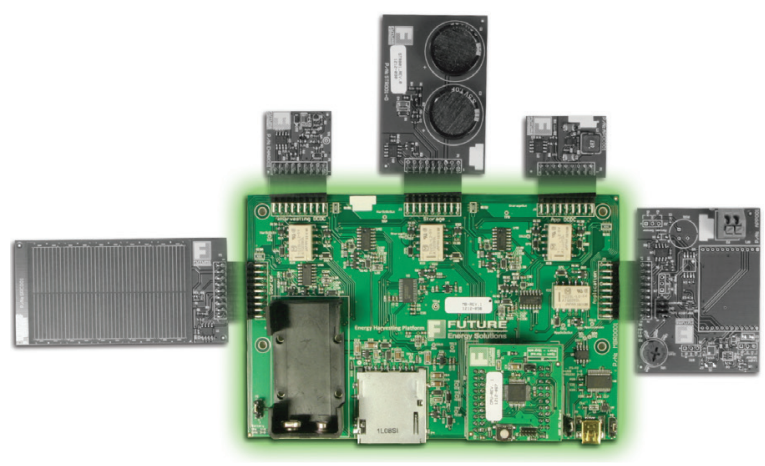

Fig. 3 The EH Platform from FutureElectronics. Different modules like dc-dc converters and storages are connected on fixed positions to the main board (shown in green) [www.futureelectronics.com] 


\section{Motivation and Approach}

\subsection{Power forming and reliability}

Continuous power supply is essential for most applications, like sensor nodes, because they have to measure and transfer data periodically. It is a challenging task to design such a continuous power supply based on energy harvesting. Keep in mind that the output power $P_{E H}$ of an energy harvester fluctuates, whereas the load shows a well-known power profile $P_{\text {load }}$. Only a micro energy management with an intermediate storage can accomplish this task [12]. A further issue is the voltage level of the harvester $V_{E H}$ which doesn't match the voltage of a typical load $V_{\text {load }}$. Consequently, the following equations hold true:

$$
\begin{aligned}
P_{E H} & \neq P_{\text {load }} \\
V_{E H} & \neq V_{\text {load }}
\end{aligned}
$$

The reliability $\rho$ of a self-powered system can be defined as the ratio between the number of jobs done (\#jobs $\left.{ }_{\text {done }}\right)$ and the number of jobs scheduled $\left(\# j o b s_{\text {scheduled }}\right)$, as shown in the following equation:

$$
\text { reliability: } \rho=\frac{\# \text { jobs }}{\# \text { done }}
$$

\subsection{Aim and Approach}

The aim of our research is to understand and to improve the reliability (as defined in Eq. 3) of selfpowered systems by means of finding the optimal energy management. Our approach presented in this paper is to develop and evaluate a construction kit to freely configure and test various managements structures. Further research will focus on the dimensioning and benchmarking of different structures.

Our approach is detailed into three steps

1. Propose a general management structure and systematically identify building blocks

2. Develop hardware modules for each building block and interconnect them with a base board

3. Prove the feasibility of the kit with measurements of the individual modules and the parasitic effects.

\section{General structure of micro energy managements}

In figure 4, we propose a general energy management structure. For the sake of simplicity, we show only one harvester, one storage and one load. The concept can be extended by multiplying the adjacent blocks of the Energy Supervisor or omitting some blocks. Thus the structure can be applied to every energy management the authors can think of. It is possible that a commercial solution could cover more than one building block, e.g. energy extraction and energy supervision.

\subsection{Identify building blocks}

The Energy Generator is responsible for collecting energy (no matter in which form) and to convert it into electrical power. Energy Extraction is essential to extract the unregulated power provided by the energy generator in the most efficient way. Impedance matching realized by Maximum power point tracking (MPPT) can be realized here. The Energy Supervisor then controls the energy flow to a load or a storage. It monitors voltage levels at different nodes in the system and activates certain power paths. To supply power with a constant regulated voltage, the Energy Supply is needed. Charge Control is mandatory to access an energy storage. It monitors the charge procedure and keeps track of overvoltage and undervoltage conditions. The Load represents the power consumer, e.g., a sensor, a microcontroller and a radio transceiver.

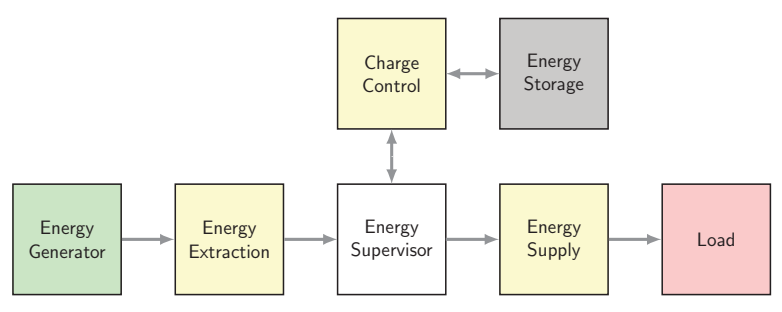

Fig. 4 General structure of energy managements

\subsection{Realizing the building blocks}

The yellow building blocks are usually realized by dc-dc converters or low drop-out regulators (LDOs). No matter if switched mode (dc-dc) or linear (LDO), every converter features a kind of regulation (input/output and current/voltage) which is presented next:

Energy Extraction requires converters which regulate the input voltage. The input voltage is wellsuited to be controlled by MPP tracking according to the fractional open-circuit voltage method (FOCV) or the perturb and observe (P\&O) method [13].

Energy Supply requires power converters which regulate the output voltage to provide e.g. 3.3 V.

For Charge Control, power converters are used which regulate the input or output current. To realize a full CCCV-charging scheme (constant current constant voltage), a voltage limiting feature is implemented as well [14]. 


\section{The construction kit: From building blocks to Modules}

\subsection{Standard module}

There are roughly about 100 integrated circuits (ICs) commercially available, which can be used in a micro energy management circuit. It is difficult to elaborate on these ICs thoroughly, because the evaluation boards of the ICs are not standardized. To overcome this issue, we have defined our own standard layout as shown in Fig. 5. One module holds all necessary components, which are (besides the IC) all necessary passive components like resistors, capacitors and inductors. This approach allows us to think in a more structured way (in building blocks) without struggling with the specific layout considerations of each IC. So far, we have managed to fit any energy management component on such a small module, like a dc-dc converter, a power switch or an energy storage.

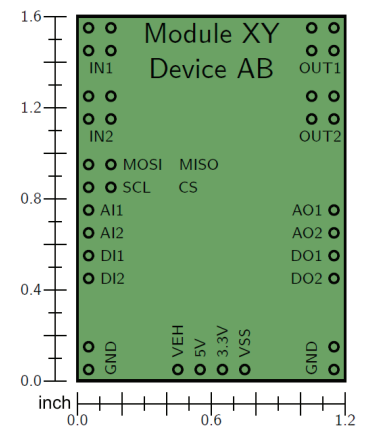

Fig. 5 Standardized module layout to hold one energy management sub-circuitry, measuring only 1.6" × 1.2"

\subsubsection{Power path}

Even though commercial ICs offer a lot of features, inputs and outputs for power can be identified and have fixed positions on every module (IN1, IN2, OUT1, OUT2, GND). Theses power ports are connected through the main board (see next section) to build up the whole power management circuitry. A four-pin connection reduces the series resistance and gives the system a mechanically reliable connection, which is necessary for long-term experiments.

\subsubsection{Control signals and further develop- ments}

Additional control inputs and outputs (analog as well as digital) are routed to the sides of the modules. They allow access to features like chip enable and under-voltage lockout (UVLO) by use of 0.1 " jumper wires. Different modes of operation of the individual chips are realized with jumpers on the module. For further developments, there is a power supply ( $5 \mathrm{~V}$, $3.3 \mathrm{~V}$ and $-1.5 \mathrm{~V}$ ) and a serial interface available.

\subsection{Main board}

Figure 6 shows the main board. It is designed to hold up to 8 modules in two rows and thus allows for multi-energy harvesting and multi-load supply.

We installed banana plugs on the edges of the board to easily connect various harvesters and loads. In the figure, a basic setup of the bq25570 dc-dc converter, a supercap and a dummy load is shown.

The main board has conducting paths which connect the modules to each other. Figure 7 (left) illustrates how the first power rail is connected from one module to the next one (IN1, OUT1, shown in red) and furthermore, that the second power rail is separated (IN1, OUT1, shown in blue and yellow). To realize even more complicated connection structures, we have also built a power jumper wire, as shown in Fig. 7 (right).

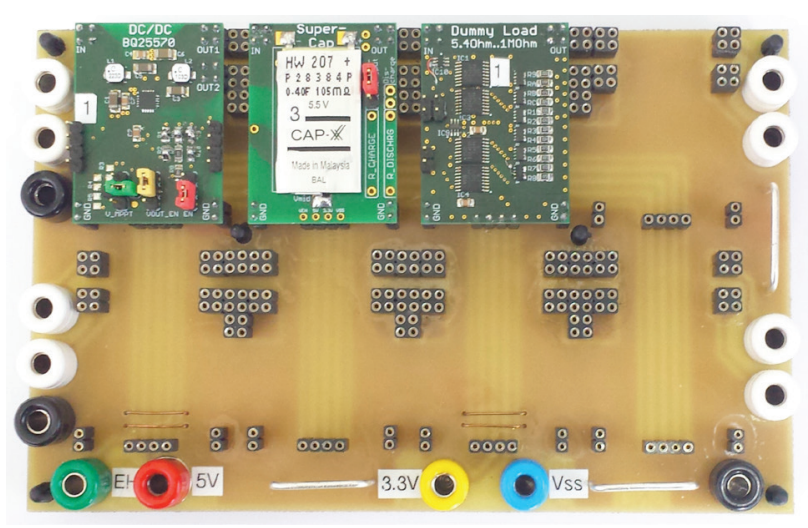

Fig. 6 Two rows of four modules each can be plugged into the main board. The inputs and outputs are accessible by the white banana plugs.

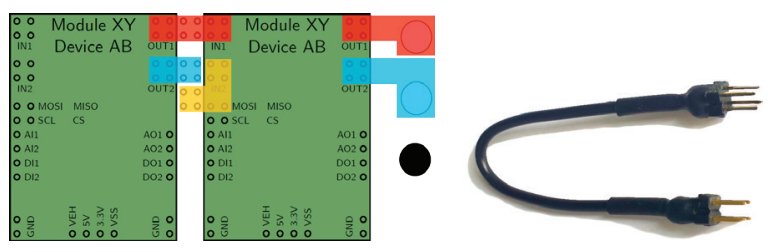

Fig. 7 left: module interconnection with two power rails; right: power jumper wire with very low contact resistance $(11 \pm 0.2 \mathrm{~m} \Omega$, including female headers) 


\subsection{Realized modules}

Table 1 summarizes all modules built so far. The first section shows all power converter modules, the second section energy supervisors, and the last section shows energy storages and a dummy load.

Table 1 Overview of all modules currently available in the construction kit

\begin{tabular}{ll}
\hline module & dedicated function \\
\hline ADP5090 & dc-dc, $V_{\text {in }}$ regulated, MPPT by FOCV \\
BQ25570 & dc-dc, $V_{\text {in }}$ regulated, MPPT by FOCV \\
SPV1040 & dc-dc, $V_{\text {in }}$ regulated, MPPT by P\&O \\
LTC3125 & dc-dc, $I_{\text {in }}$ regulated, $0.2 \mathrm{~A}$ to $1 \mathrm{~A}$ \\
LT3109 & ac-dc, $V_{\text {startup }}=20 \mathrm{mV}$, for TEGs \\
\hline TPS3839x & two voltage supervisors 2.6 V and 3.1 V \\
ADG888 & analog switch matrix $(5.5 \mathrm{~V}, 0.8 \mathrm{~A})$ \\
TPS2421 & load switch $(20 \mathrm{~V}, 5 \mathrm{~A})$ \\
LT6656 + & voltage reference and comparator \\
TLV3691 & on one module $\left(I_{\mathrm{q}}=850 \mathrm{nA}\right.$ and $\left.75 \mathrm{nA}\right)$ \\
\hline SuperCap & $C=0.18,0.3$ and $0.8 \mathrm{~F}$ from Cap-XX \\
DummyLoad & prog. resistor array $12.5 \mathrm{~m} \Omega$ to $1 \mathrm{M} \Omega$ \\
\hline
\end{tabular}

In the following, we will give more details about certain modules: figure 8 shows the ADP5090 and the BQ25570 module. Both converters feature two outputs: the first is meant to connect a super capacitor, where the second one is a voltage controlled output. The pin Al1 is the fractional open-circuit voltage which is acquired by the dc-dc controller. With the jumpers $(\mathrm{J} 1 \ldots \mathrm{J} 3)$ or the digital inputs (DI1 and DI2) the FOCV ratio can be chosen and an undervoltage feature can be enabled.

The switch matrix shown in Fig. 9 is built by two ADG888 from Analog Devices. The module features two inputs and two outputs. By means of the
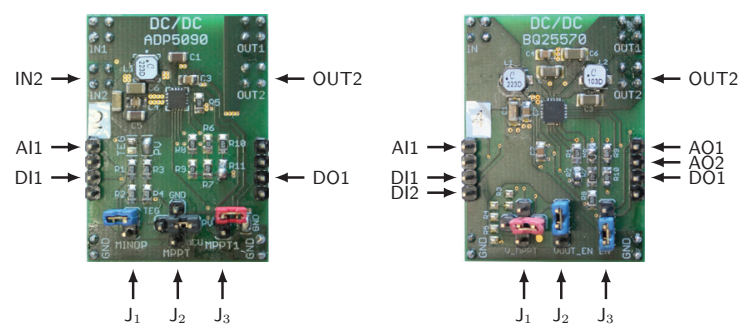

Fig. 8 left: ADP5090 module; right: BQ25570 module
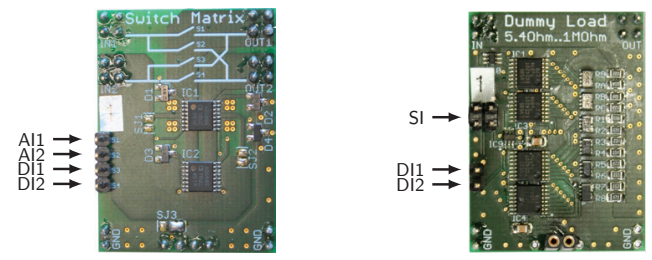

Fig. 9 left: Switch matrix; right: dummy load module four bidirectional switches, every input can either be connected to every output or left floating. On the right of Fig. 9 the dummy load is depicted. It contains a 16-Bit shift register to address 16 precision resistors $(0.1 \%)$ via MOSFET transistors. The values are $12.5 \Omega, 25 \Omega, 50 \Omega, 100 \Omega, 220 \Omega, 470 \Omega$, $1 \mathrm{k} \Omega, 2.2 \mathrm{k} \Omega, \ldots 470 \mathrm{k} \Omega$ and $1 \mathrm{M} \Omega$.

\section{Experimental results}

It is important that implementing the power management ICs on our modules has no effect on the performance and the behavior in any manner. This is why be thoroughly tested each module and compared the results to the reference data (datasheet).

\subsection{Validation of the power converter modules}

The main parameters of interest of power converters are the start-up voltage and the efficiency. We use the Keithley SourceMeter 2400 as a current source and the B\&K Precision 8600 as a current sink. The current and voltage at the input and output were measured with four Agilent 43301A. We carried out an automated measurement using LabVIEW by sweeping current and voltage for all power modules. Exemplary, we show the results for the ADP5090 and BQ25570 in Fig.10 to 13. The reference data is always given as a blue line and the deviation is shown in a separate chart on the right.
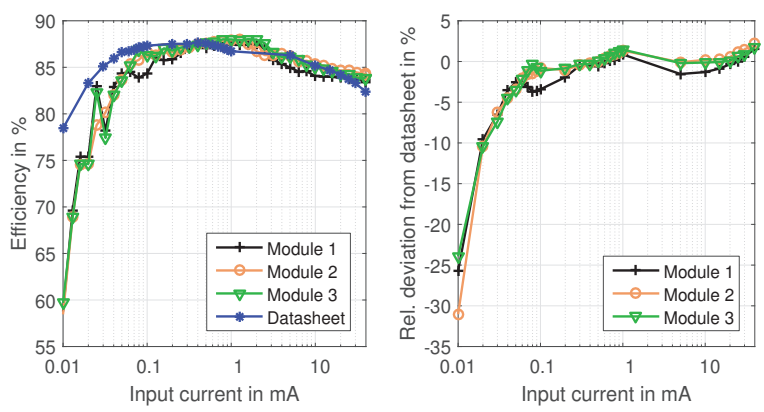

Fig. 10 ADP5090 efficiency measurement in dependence of input current (left) and relative deviation (right)
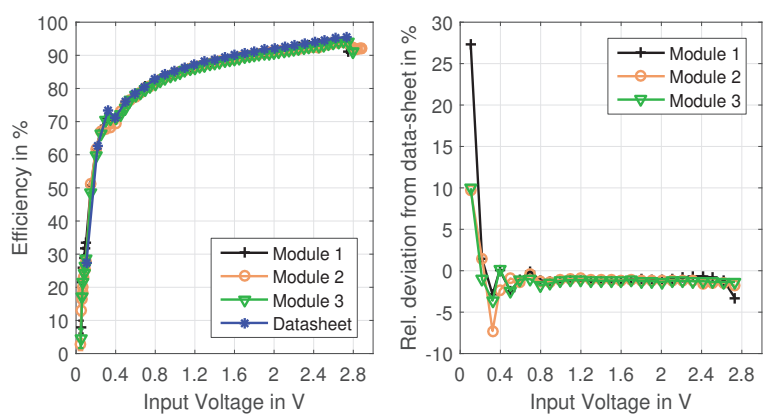

Fig. 11 ADP5090 efficiency measurement in dependence of input voltage (left) and relative deviation (right) 



Fig. 12 BQ25570 efficiency measurement in dependence of input current (left) and relative deviation (right)
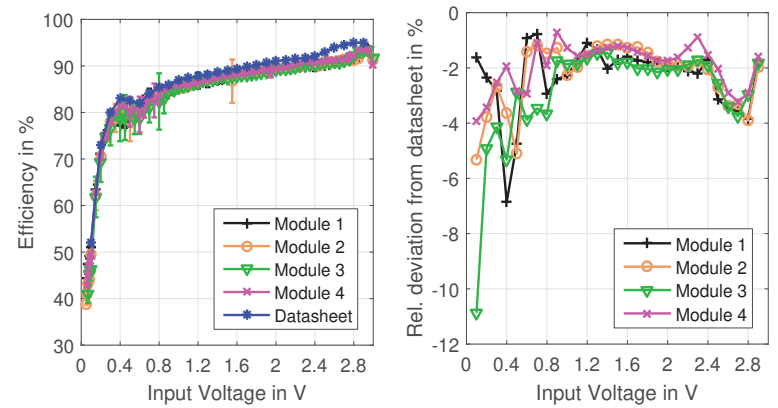

Fig. 13 BQ25570 efficiency measurement in dependence of input voltage (left) and relative deviation (right)

\subsection{Validation of the supervisor modules}

As an example for the supervisor modules, we show the validation of the switch matrix module. We measured the on-resistance $R_{\mathrm{on}}$ of switch S1 and S2 (IN1-to-OUT1 and IN1-to-OUT2) as shown in Fig. 14. We conducted a four-wire measurement at a constant current of $100 \mathrm{~mA}$. Further, we measured the off-resistance $R_{\text {off }}$ to be greater then $5 \mathrm{M} \Omega$ for all input voltages.
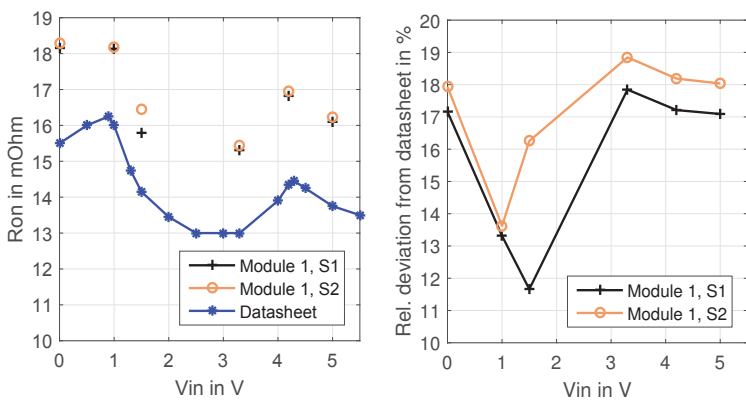

Fig. 14 On-resistance $(S 1, S 2)$ of the switch matrix

\subsection{Validation of the Supercap}

The super cap module (shown in Fig. 6; 2nd module) holds the SC HW 207 from the company CAP-XX with a nominal capacity of $0.4 \mathrm{~F}$. We charged the capacitor with a constant current of $3 \mathrm{~A}$ up to $5.3 \mathrm{~V}$ and left the output floating at time $t=0$. The data was



Fig. 15 Charging and relaxation of a $0.4 \mathrm{~F}$ super cap, measured three times, in comparison with a simulation

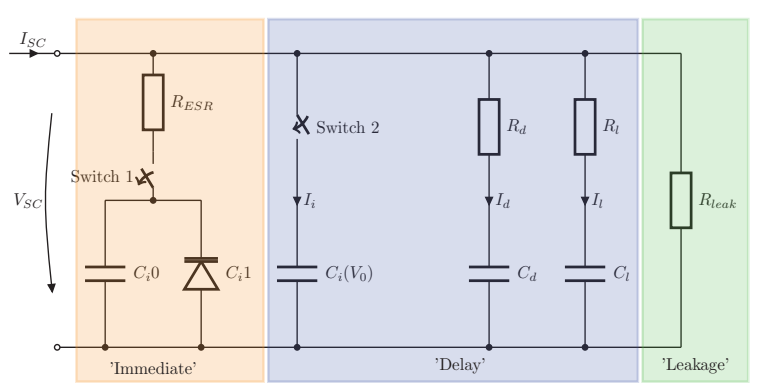

Fig. 16 Proposed supercap equivalent model. Switches separate between the charging and discharging process

acquired by a NI myDAQ (16 Bit ADC resolution, $200 \mathrm{kS} / \mathrm{s}$ ). In Fig. 15 we can clearly see the voltage drop, due to the internal resistance and charge redistribution effect. Based on [15] we developed a equivalent circuit of the super capacitor as shown in Fig. 16. We introduced two switches to separate between the charging and the discharging process. If the voltage reaches $5.3 \mathrm{~V}$ switch 1 opens and switch 2 closes simultaneously.

\subsection{Validation of the dummy load}

The dummy load can generate 65.536 parallel resistors combinations as explained in Sec. 5.3. For validation it is sufficient to apply a one-hot coding and thus only measure one precision resistor at a time. We carried out the measurement at 8 temperatures ranging from $-10^{\circ} \mathrm{C}$ to $60^{\circ} \mathrm{C}$ as shown in Fig. 17.

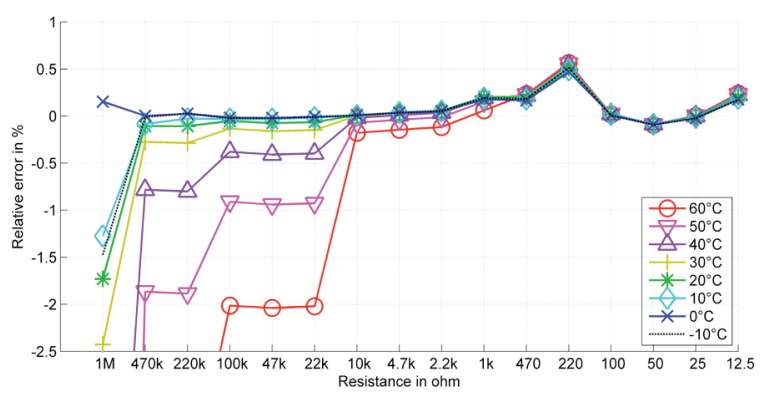

Fig. 17 Measured relative error of the 16 individual resistors of the dummy load at 8 different temperatures 


\subsection{Parasitic effects of modules}

Besides the modules, the main board itself and the interconnection may interfere the measurement setup. To clarify the order of magnitude of these effect we have done a short evaluation on the PCB traces and interconnections. Table 2 summarizes the results. The contact resistance was measured by applying a four wire measurement with a current of $1 \mathrm{~A}$. We used a Fluke 87 III multimeter to measure the differential voltage of the four wire measurement, and furthermore the conductance and the capacitance.

Table 2 Overview of parasitic effects of the main board and the module interconnection

\begin{tabular}{ll}
\hline Quantity & result \\
\hline Contact resistance, module-board & $5 \pm 0.1 \mathrm{~m} \Omega$ \\
Power jumper wire, board-board & $11 \pm 0.2 \mathrm{~m} \Omega$ \\
Isolation resistance (IN1 to GND) & $>100 \mathrm{G} \Omega$ \\
Capacitance (IN1 to GND) & $<10 \mathrm{pF}$ \\
Capacitance (IN1 to IN2) & $<10 \mathrm{pF}$ \\
\hline
\end{tabular}

\subsection{Discussion of the measurements results}

The efficiency results of the power converter are in good accordance with the datasheet values. For lower input currents and voltages, the relative error seems to be is higher due to the chosen presentation. The validation of the switch matrix module shows an on-resistance which is about $18 \%$ higher than the datasheet value. This could be due to the PCB traces and interconnections. The charging behavior of the supercapacitor matches very well to our simulated model. The dummy load shows significant deviation from the expected $0.1 \%$ precision of the resistors. For the high-ohmic resistors, the negative relative error is due to the leakage current of the transistors. For the low-ohmic resistors, the positive relative error is due to the on-resistance of the transistors.

The measurement of the parasitics of modules and mainboard clearly show, that these effects are negligible. Further keep in mind, that all critical circuit paths are only on the modules, and thus the module interconnection dose not influence the function of these building blocks.

\section{Conclusion and Outlook}

We proposed a generic energy management which can be applied to a large variety of self-powered systems. Based on the identification of building blocks, we defined and built a novel modular construction kit. We built all necessary blocks like power converters, energy supervisors, energy storages and a load as modules. A detailed evaluation shows, that all modules work within their specification and that the measurement kit itself has no relevant influence on the measurement. The platform is very flexible and can be kept up-to-date very easily.

Our tool offers us a new approach to thoroughly investigate energy managements for further research. Regarding development of more modules we will focus on measurement modules and thus, enhance the system with self-monitoring capabilities. This will make the need of laboratory measurement gear unnecessary and will allow for long-term measurements outdoors.

\section{Acknowledgments}

This work was financially supported by the Ministry of Science, Research and Arts of BadenWürttemberg, Germany (Graduiertenkolleg DENE). A very special thank goes to Emma Walton.

\section{References}

[1] P. Sitka, P. Corke, L. Overs, P. Valencia, and T. Wark, "Flecka platform for real-world outdoor sensor networks," in Intelligent Sensors, Sensor Networks and Information, 2007. ISSNIP 2007. 3rd International Conference on. IEEE, 2007, pp. 709-714.

[2] H. Park, J. Friedman, P. Gutierrez, V. Samanta, J. Burke, and M. Srivastava, "Illumimote: Multimodal and high-fidelity light sensor module for wireless sensor networks," Sensors Journal, IEEE, vol. 7, no. 7, pp. 996-1003, July 2007.

[3] J. Kim and J. P. Lynch, "Experimental analysis of vehiclebridge interaction using a wireless monitoring system and a two-stage system identification technique," Mechanical Systems and Signal Processing, vol. 28, pp. 3-19, 2012.

[4] A. Zerger, R. V. Rossel, D. Swain, T. Wark, R. Handcock, V. Doerr, G. Bishop-Hurley, E. Doerr, P. Gibbons, and C. Lobsey, "Environmental sensor networks for vegetation, animal and soil sciences," International Journal of Applied Earth Observation and Geoinformation, vol. 12, no. 5, pp. 303 316, 2010.

[5] R. Vullers, R. Schaijk, H. Visser, J. Penders, and C. Hoof, "Energy harvesting for autonomous wireless sensor networks," Solid-State Circuits Magazine, IEEE, vol. 2, no. 2, pp. 29-38, Spring 2010.

[6] A. S. Weddell, G. V. Merrett, T. J. Kazmierski, and B. M. Al-Hashimi, "Accurate supercapacitor modeling for energy harvesting wireless sensor nodes," Circuits and Systems II: Express Briefs, IEEE Transactions on, vol. 58, no. 12, pp. 911-915, 2011.

[7] G. V. Merrett and A. S. Weddell, "Supercapacitor leakage in energy-harvesting sensor nodes: Fact or fiction?" in Networked Sensing Systems (INSS), 2012 Ninth International Conference on. IEEE, 2012, pp. 1-5.

[8] J. Varley, M. Martino, S. Poshtkouhi, and O. Trescases, "Battery and ultra-capacitor hybrid energy storage system and power management scheme for solar-powered wireless sensor nodes," in IECON 2012-38th Annual Conference on IEEE Industrial Electronics Society. IEEE, 2012, pp. 48064811. 
[9] F. Ongaro, S. Saggini, and P. Mattavelli, "Li-ion batterysupercapacitor hybrid storage system for a long lifetime, photovoltaic-based wireless sensor network," IEEE Transactions on Power Electronics, vol. 27, no. 9, pp. 3944-3952, Sept 2012.

[10] W. Wang, V. Cionca, N. Wang, M. Hayes, B. O'Flynn, and C. O'Mathuna, "Thermoelectric energy harvesting for building energy management wireless sensor networks," International Journal of Distributed Sensor Networks, 2013.

[11] J. Nagel, Neues Konzept für die bedarfsgerechte Energieversorgung des Künstlichen Akkommodationssystems, ser. Schriftenreihe des Instituts für Angewandte Informatik, Automatisierungstechnik am Karlsruher Institut für Technologie. KIT Scientific Publishing, Karlsruhe, 2012.

[12] V. Raghunathan, A. Kansal, J. Hsu, J. Friedman, and M. Srivastava, "Design considerations for solar energy harvesting wireless embedded systems," in Proceedings of the 4th international symposium on Information processing in sensor networks. IEEE Press, 2005, p. 64.

[13] D. Dondi, A. Bertacchini, D. Brunelli, L. Larcher, and L. Benini, "Modeling and optimization of a solar energy harvester system for self-powered wireless sensor networks," Industrial Electronics, IEEE Transactions on, vol. 55, no. 7, pp. 2759-2766, 2008.

[14] M. Einhorn, F. Conte, C. Kral, C. Niklas, H. Popp, and J. Fleig, "A modelica library for simulation of elecric energy storages," in Proceedings of the 8th International Modelica Conference, 2011.

[15] J. Lindenmaier, "Untersuchung eines von Doppelschichtkondensatoren unterstuetzten Zweispannungsbordnetzes für Mikro-Hybrid-Fahrzeuge," Ph.D. dissertation, University Ulm, 2010 